\title{
PUBLICAÇÃO DIGITAL SOBRE A EXTENSÃO UNIVERSITÁRIA NA UNIVILLE
}

\section{DIGITAL PUBLICATION ABOUT THE UNIVERSITY EXTENSION IN UNIVILLE}

\author{
Jenifer Ramos ${ }^{1}$, Bach. \\ Roy Ristow Wippel Schulenburg², M.Sc. \\ (1) Universidade da Região de Joinville - UNIVILLE \\ e-mail: jenifer.ramos@live.com \\ (2) Universidade da Região de Joinville - UNIVILLE \\ e-mail: royzera@gmail.com
}

Palavras-chave: design de interface, design de interação, publicação digital

\begin{abstract}
O presente artigo apresenta a síntese de um trabalho de conclusão de curso que consiste no desenvolvimento de uma publicação digital a partir de um processo participativo incorporando questões de acessibilidade. Essa publicação tem como premissa apresentar a extensão universitária no contexto de atuação da UNIVILLE, tal como programas que representam as ações da atividade extensionista da instituição nas diferentes áreas do conhecimento. Esse trabalho se fez a partir de um processo colaborativo, o diagrama do Diamante Duplo do Design Thinking, cujo escopo é estruturado em quatro etapas: Descobrir, na qual fez-se uma pesquisa bibliográfica exploratória; Definir, na qual convergiu-se informações com uma coleta e análise de dados; Desenvolver, na qual fez-se protótipos que foram testados, refinados e validados de maneira colaborativa; e "Deliverar", na qual fez-se a exposição da solução final. O trabalho resulta no design da interface de uma publicação digital interativa com multimídia acessível, construída de maneira participativa, que intenta apresentar a atividade extensionista da UNIVILLE.
\end{abstract}

Key-words: interface design, interaction design, digital publication

The present work consists in the research, data collect, conceptualization, development, test and validation of a digital publication which premise is to present the university extension into UNIVILLE's actuation context, like the institution's programs and projects of extension that represent their actions in the different areas of knowledge. The methodological process is based in the Design Thinking Double Diamond diagram, dividing the work in four steps: Discover, where an explanatory research were done; Define, where data and information were converged; Develop, where prototypes were done, tested, readjusted and refined in a participatory way; and Deliver, where the final solution were achieved. The work results in a multimedia interactive digital publication that intends to spread and disseminate UNIVILLE's extensionist activity, built in collaboration with potential users and other stakeholders. 


\section{$16^{\circ}$ \\ ERGODESIGN USIHC CINAHPA}

\section{Introdução}

O trabalho consiste no desenvolvimento de uma publicação digital que trata da atividade extensionista da UNIVILLE, seus fundamentos, objetivos, relevância em aspectos sociais e acadêmicos e alguns dos seus programas atuantes em diferentes áreas do conhecimento. A publicação pode ser visualizada em diferentes dispositivos digitais, ela contempla questões de acessibilidade e foi construída de maneira participativa.

A estrutura desse trabalho baseou-se no diagrama do Diamante Duplo do Design Thinking, interpretado por Pinheiro e Alt (2011), processo colaborativo dividido em quatro etapas, como apresenta a Figura 1.

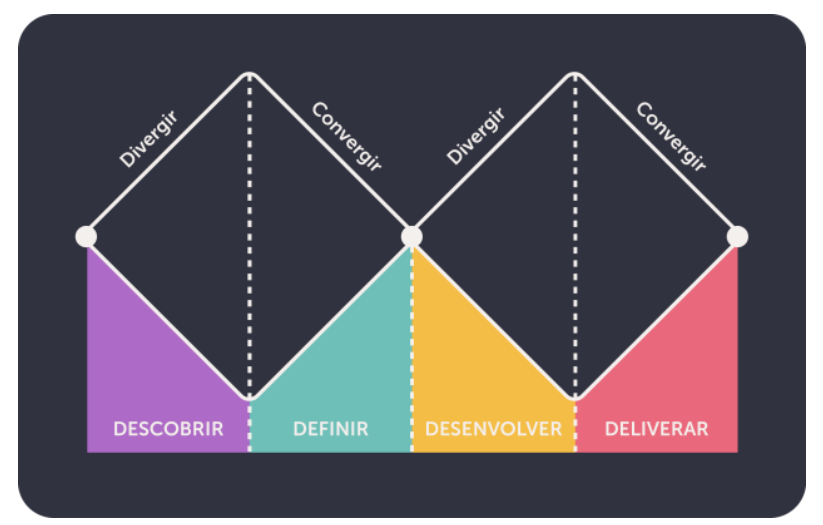

Figura 1 - Diagrama do Diamante Duplo Fonte: Pinheiro e Alt (2011) adaptados

\section{Descobrir}

Em Descobrir pretende-se, segundo Pinheiro e Alt (2011), expandir informações ao divergir do centro do trabalho para o seu redor. Portanto, nessa etapa, descobriu-se conhecimentos através de uma pesquisa exploratória em materiais bibliográficos coerentes e atualizados. Foram abordados, assim, os seguintes assuntos: extensão universitária; publicações digitais; grid e diagramação digital; tipografia digital; e design de interação.

Em extensão universitária, descobriu-se conhecimentos sobre a extensão a partir de diferentes concepções, com foco na abordagem em $16^{\circ}$ Ergodesign - Congresso Internacional de Ergonomia e Usabilidade de Interfaces Humano Tecnológica: Produto, Informações Ambientes Construídos e Transporte

$16^{\circ}$ USIHC - Congresso Internacional de Ergonomia e Usabilidade de Interfaces Humano Computador

CINAHPA | 2017 - Congresso Internacional de Ambientes Hipermídia para Aprendizagem. instituições de ensino superior comunitárias, como a UNIVILLE. Além disso, foram reunidas informações sobre a UNIVILLE e sua atuação na extensão universitária. Em publicações digitais averiguou-se dispositivos capazes de interpretar publicações digitais, os formatos mais populares de publicações e suas diferentes características de acordo com as atuais possibilidades, uma vez que as tecnologias estão em constante inovação.

Posteriormente, buscou-se informações acerca do que é e os tipos de grid e as heurísticas relacionadas à diagramação de interfaces, do tipo de imagem e suas características para melhor visualização em telas ao comportamento dos blocos de texto na interface. A partir disso, ampliou-se a pesquisa para tipografia, seus tipos, construção da fonte e seu comportamento na tela entre outras informações. Por fim, descobriu-se conhecimentos sobre design de interação incorporando design de interface, usabilidade, acessibilidade e experiência do usuário.

As principais referências para a pesquisa bibliográfica foram, tratando-se de extensão universitária, Jezine (2004) e Síveres (2013); tratando-se de publicações digitais, Monteiro (2013) e Sehn (2014), além de desenvolvedores de formatos de publicações, como Adobe (2016); tratando-se de grid e diagramação digital, Lupton (2015) e Puppi (2014); tratando-se de tipografia digital Henestrosa et al. (2014) e Lupton (2015); e tratando-se de design de interação Cooper et al. (2014) e Rogers et al. (2013).

\section{Definir}

Para Pinheiro e Alt (2011), nessa fase do processo intenta-se constatar o significado e a relevância dos conhecimentos anteriormente descobertos. Assim foi definido, de maneira mais objetiva, a abordagem do tema do trabalho e as informações que farão parte do objeto resultante desse processo.

\subsection{Entrevistas semisestruturadas}

Fez-se uma coleta de informações relacionadas à extensão na UNIVILLE através de entrevistas semiestruturadas. Para essa coleta foram entrevistados quatro docentes da UNIVILLE que 


\section{$16^{\circ}$ \\ ERGODESIGN USIHC CINAHPA}

participaram na execução e/ou coordenação de programas de extensão da instituição.

Tratou-se, assim, do Programa de Literatura Infantil Juvenil - PROLIJ; do Programa Estruturante de Empreendedorismo - PEE; do Programa Matur(a)idade na UNIVILLE; e do Farmácia Escola. Estes programas expressam na prática as ações da extensão na instituição em diferentes áreas do conhecimento.

\subsection{Diagrama de Afinidade}

Com o diagrama de afinidade sistematizou-se o que foi coletado nas entrevistas baseando-se em conexões por afinidade, proximidade, dependência ou similaridade utilizando cartões de insights que, por sua vez, são cartões que sintetizam os tipos de informações que foram coletadas (VIANNA et al., 2012). Com o Diagrama de Afinidade foi possível sintetizar e sistematizar as informações coletadas com as entrevistas para, então, melhor organizá-las e dispô-las na publicação digital.

\subsection{Mood boards}

Reunindo diferentes materiais institucionais da UNIVILLE elaborou-se alguns mood boards para explanar a sua identidade visual. Uma vez que a publicação digital desenvolvida está ligada à instituição, desenvolveu-se tais mood boards com a premissa de manter a publicação visualmente harmônica com a linguagem gráfica da UNIVILLE. Assim, foram desenvolvidos três mood boards, sendo o primeiro com flyers digitais, o segundo, apresentado pela figura 2, com cartilhas e guias institucionais e o terceiro com capas e contracapas destas mesmas cartilhas e guias.

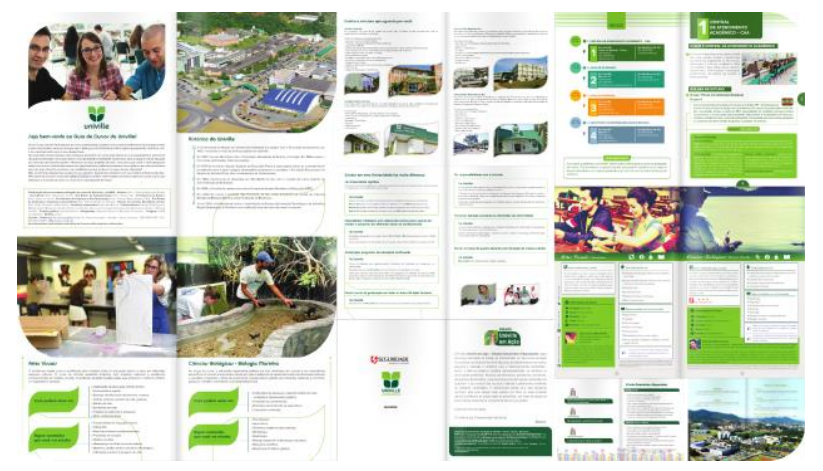

$16^{\circ}$ Ergodesign - Congresso Internacional de Ergonomia e Usabilidade de Interfaces Humano Tecnológica: Produto, Informações Ambientes Construídos e Transporte

$16^{\circ}$ USIHC - Congresso Internacional de Ergonomia e Usabilidade de Interfaces Humano Computador

CINAHPA | 2017 - Congresso Internacional de Ambientes Hipermídia para Aprendizagem.

Figura 2 - Mood board com materiais da UNIVILLE Fonte: Primária (2016)

Os mood boards originaram várias informações, cuja sintetização foi feita através de uma Nuvem de Palavras, apresentada pela figura 3.

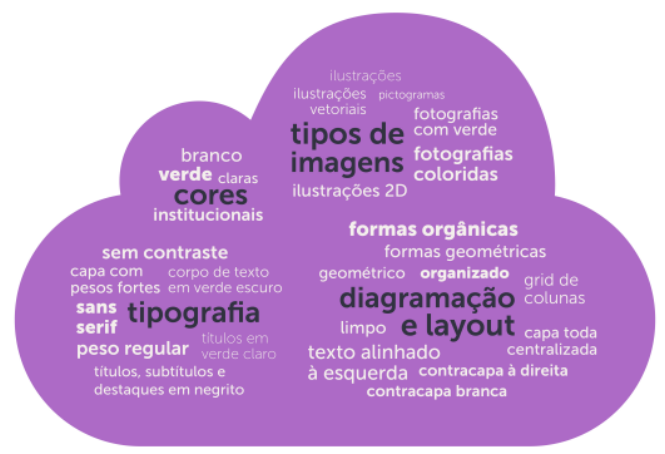

Figura 3 - Nuvem de Palavras

Fonte: Primária (2016)

\subsection{Mapa conceitual}

Comumente utilizado no processo do Design Thinking, o mapa conceitual tem como premissa auxiliar a definir e conectar informações (VIANNA et al., 2012). A partir das informações até então reunidas, elaborou-se um mapa conceitual a fim de convergir tudo que foi pesquisado e coletado de maneira estrita ao que será aplicado no trabalho em si. O mapa é apresentado pela figura 4 .

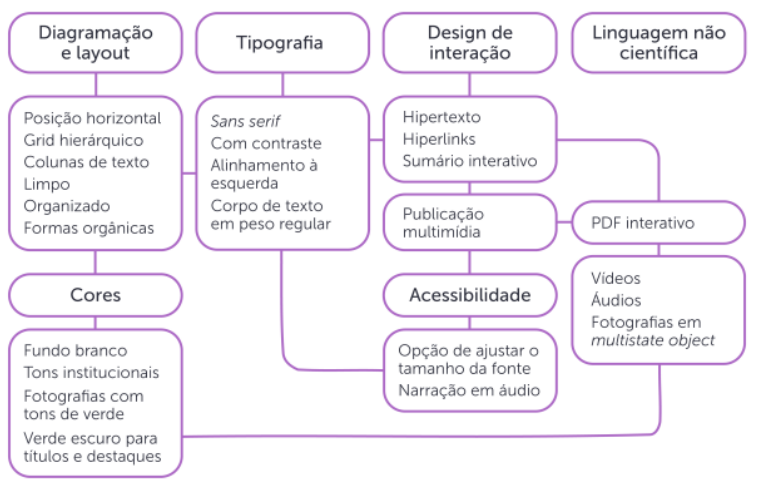

Figura 4 - Mapa conceitual Fonte: Primária (2016)
Realização:
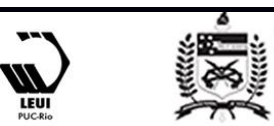


\section{$16^{\circ}$ \\ ERGODESIGN USIHC CINAHPA}

Com essa ferramenta pôde-se filtrar e visualizar as informações pertinentes à interface digital descobertas e definidas dispensando, assim, uma etapa de geração de alternativas.

\subsection{Card sorting}

Com o objetivo de definir os conteúdos da publicação, tal como ordená-los como em um sumário, o card sorting foi aplicado com uma docente da área de extensão da UNIVILLE. Uma vez que a publicação desenvolvida trata de um tema pouco disseminado, a extensão, fez-se essa definição de conteúdo de maneira colaborativa com uma pessoa diretamente envolvida na extensão, considerando sua percepção quanto aos tópicos mais pertinentes e relevantes no entendimento da atividade extensionista e na forma com que eles se conectam. Foram criados cartões baseados nos assuntos em relação à extensão que foram explorados ao descobrir conhecimentos, na primeira etapa do trabalho, os quais a participante organizou. Os cartões em lilás representam os originalmente entregues a participante, enquanto os em turquesa são os que ela acrescentou.

\section{Desenvolver}

Com a expansão de possibilidades estabelecida ao divergir para descobrir novos conhecimentos, tal como com a coleta e análise de dados concluída ao convergir para definir informações, passa-se para a etapa de construção do objeto ao divergir para desenvolver a solução do problema de pesquisa.

Conforme Pinheiro e Alt (2011), embora o processo do Diamante Duplo tenha uma lógica linear, devido à abordagem colaborativa, ele é livre para seguir uma natureza interativa e/ou cíclica, com o uso de ferramentas e/ou métodos colaborativos e participativos com etapas de teste, refinamento e validação.

Nesse trabalho, essa progressão cíclica e interativa evidencia-se na presente etapa de Desenvolver, ao envolver potenciais usuários e outros stakeholders na evolução do desenvolvimento da publicação digital, a fim de moldá-la conforme as $16^{\circ}$ Ergodesign - Congresso Internacional de Ergonomia e Usabilidade de Interfaces Humano Tecnológica: Produto, Informações Ambientes Construídos e Transporte

$16^{\circ}$ USIHC - Congresso Internacional de Ergonomia e Usabilidade de Interfaces Humano Computador

CINAHPA | 2017 - Congresso Internacional de Ambientes Hipermídia para Aprendizagem. necessidades, percepções e interesses de tais colaboradores. Dessa forma, a abordagem cíclica se fez através de uma sucessão de prototipações e validações da publicação, a qual foi desenvolvida, testada pelo stakeholder, validada sob acompanhamento e, a partir dos feedbacks do mesmo, refinada para ser testada novamente até que obteve-se um resultado satisfatório.

O processo de prototipação se fez em três fases: prototipação de baixa fidelidade, na qual fez-se esboços das telas manualmente; protótipos de média fidelidade, na qual fez-se wireframes considerando informações como resolução final e tipo de conteúdo; e, por fim, protótipos de alta fidelidade, com simulação de texto e finalização de layout, os quais foram utilizados para testes com os devidos colaboradores.

Ao total foram feitos quatro testes com a prototipação para validação do projeto gráfico desenvolvido para a publicação. Os dois primeiros testes consistiram em avaliar e validar, essencialmente, o design da interface em diferentes dispositivos digitais. O terceiro teste direcionou-se ao design da interface junto à percepção e organização do conteúdo, enquanto o quarto teste buscou avaliar a experiência do usuário com o design de interação, além das percepções do design da interface como um todo, com a proposta final do projeto gráfico.

O primeiro teste foi feito com uma acadêmica do curso de Design. Através de um arquivo PDF, mesmo formato da publicação final, ela testou e avaliou os protótipos de alta fidelidade em um notebook. O segundo teste foi feito com os mesmos protótipos de alta fidelidade mas, desta vez, avaliados em um tablet. A segunda participante foi uma mestranda em Biologia. Com os feedbacks obtidos com esses dois primeiros testes, fez-se alguns refinamentos nos protótipos para, posteriormente, serem testados novamente. Com conteúdo textual legítimo, a publicação foi compartilhada com uma terceira colaboradora, uma docente envolvida na extensão na UNIVILLE. Após esses três testes, análises e validações colaborativas, fez-se uma proposta final com as interações funcionais a qual foi testada com um 


\section{$16^{\circ}$ \\ ERGODESIGN USIHC CINAHPA}

quarto e último stakeholder, em um computador de mesa. A última prototipação apresenta recursos de interação como o sumário interativo, vídeos incorporados, exibição de múltiplas imagens e recursos de acessibilidade.

Foram testadas, ao total, oito telas com os colaboradores: tela somente com texto; tela com texto + imagens; somente imagens; texto + vídeo; texto + vídeo + imagens; somente vídeo; sumário; e tela de apresentação. Conforme feedbacks, foi descontinuada a tela de texto + vídeo + imagens, pois constatou-se um excesso de informação que confundia o usuário.

A interface possui um menu com recursos de aumento do tamanho da tipografia e narração em áudio. Como pesquisado em Descobrir, conforme Cooper et al. (2014), recursos de acessibilidade devem fazer parte da interface de modo visível, porém não gritante. Devem-se comportar como uma opção que o usuário que precisa facilmente identifica, e o usuário que não precisa não se incomode em tê-la na interface (COOPER et al., 2014). Nos testes, os recursos de acessibilidade foram validados, inclusive, com os usuários que não possuem deficiência. No caso da narração em áudio, alguns usuários se interessaram pela opção para ser utilizada em momentos em que não podem lidar com a interface. Quanto à possibilidade de aumentar ou diminuir o tamanho da tipografia, usuários relataram que esse recurso também é útil em circunstâncias como cansaço noturno ou excesso de luz diurna, refletindo a interface. Nessas situações, segundo os usuários, ter a opção de ampliar a tipografia facilita a leitura. Sendo assim, constatou-se que a acessibilidade não se limita a aprimorar a usabilidade da interface para pessoa com deficiência, como também melhora a experiência dos usuários sem deficiência.

Intrinsecamente ligada à UNIVILLE a publicação possui várias características advindas da identidade visual da instituição, como o constante uso dos tons de verde da marca da UNIVILLE. Em geral, a publicação foi construída a partir de um grid hierárquico que conta com três colunas bases para as páginas que têm conteúdo textual, além de marcadores fixos. A família tipográfica selecionada foi a Source Sans. O alinhamento dos blocos de texto é justificado e os parágrafos são distintos por um recuo na primeira linha.

\section{Deliverar}

Essa etapa concentra-se na entrega de tal material através da apresentação do projeto gráfico da publicação digital.

São expostas aqui as telas que correspondem aos padrões que são repetidos no decorrer da publicação, tal como a tela do sumário, que consiste em uma unidade dentro do padrão. A publicação, que tem como premissa apresentar e disseminar a atividade extensionista da UNIVILLE, consiste em um PDF interativo projetado em resolução de tela tida como padrão. O projeto gráfico da publicação digital pode ser visualizado com a figura 5 , que apresenta as telas bases do grid.
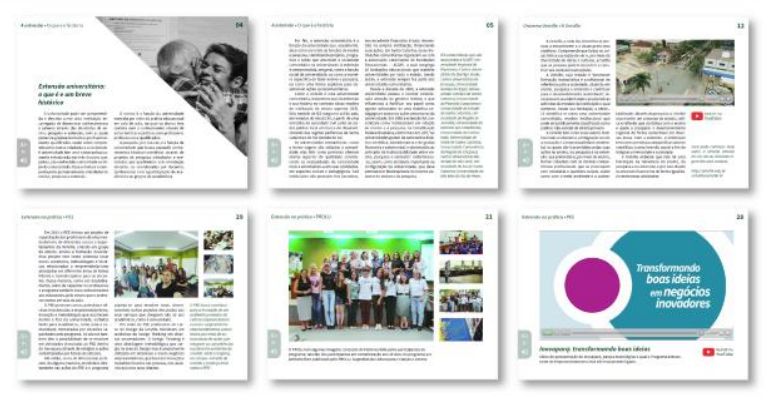

Figura 5 - Publicação digital: telas bases Fonte: Primária (2016)

Ela é apropriada para tablets, mas também pode ser proporcionalmente exibida em outros dispositivos digitais, como computadores e smartphones.

Multimídia, a publicação traz vídeos incorporados, tal como seus devidos links para serem assistidos online. Ela apresenta imagens múltiplas em uma única tela, além dos recursos de acessibilidade: narração em áudio do conteúdo e possibilidade de alterar o tamanho da tipografia, seja para maior ou menor. Ao lado direito, ela possui uma coluna com texto complementar ao principal. A interface da tela com múltiplas imagens é apresentada pela figura 6 .

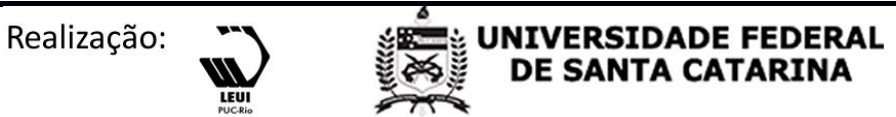


$16^{\circ}$ USIHC - Congresso Internacional de Ergonomia e Usabilidade de CINAHPA Interfaces Humano Computador

CINAHPA | 2017 - Congresso Internacional de Ambientes Hipermídia para Aprendizagem.

Extensão na prática $\cdot$ Matur(a)idade
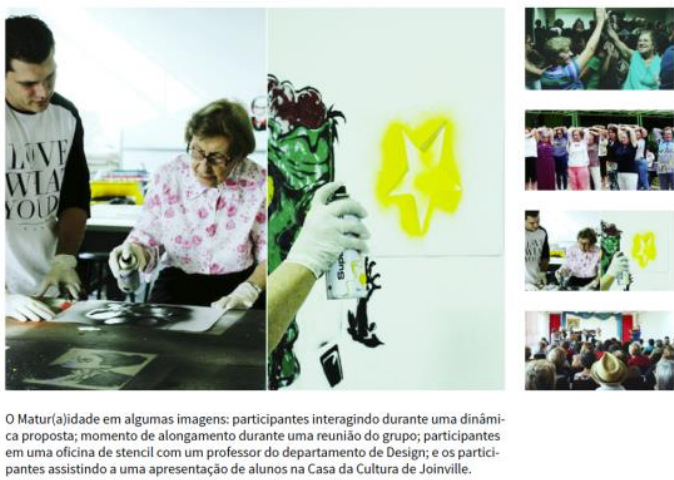

Figura 6 - Tela de imagens em destaque Fonte: Primária (2016)

A interface da publicação possui, em geral, um layout limpo, alinhado e com formas retas. $\mathrm{O}$ sumário é a uma página clara e intuitiva, além de interativa: é possível, por meio de um clique sob o nome do capítulo desejado, que o usuário seja direcionado a ele. A tela final traz links diretos pro site da UNIVILLE, assim como para algumas das suas principais redes sociais.

\section{Considerações finais}

O trabalho cuja premissa era desenvolver uma publicação digital que explane a extensão universitária na UNIVILLE teve a elaboração de tal objeto concluída. A publicação digital aborda a extensão na UNIVILLE exemplificando suas ações com alguns programas de caráter contínuo que atuam em diferentes áreas do conhecimento. Uma vez que tal objeto possa colaborar na divulgação da extensão, entende-se que podem ser geradas outras publicações digitais da mesma natureza para apresentar outros programas e projetos de extensão e, até mesmo, de pesquisa, assim como outros projetos, ações, cursos, parcerias, pesquisas e/ou áreas da universidade. A publicação contempla recursos de acessibilidade inerentes ao seu layout úteis não exclusivamente a pessoas com deficiência, como a todos os usuários em geral, nas devidas circunstâncias.

\section{BIBLIOGRAFIA}

ADOBE. Adobe PDF history. Disponível em: <http://bit.ly/PDFhistory>. Acesso em 17 de jan. de 2016.

COOPER, Alan; REIMANN, Robert; CRONIN, David; NOESSEL, Christopher. About face: the essencials of interaction design. 4. Ed. Indianápolis: Editora Wiley, 2014.

JEZINE, Edineide. As práticas curriculares na extensão universitária. In: CUNHA, Eleonora Schettini Martins Cunha; CARVALHO, Alysson Mascote (Org.). (Re)conhecer diferenças, construir resultados. Brasília-DF: Unesco, 2004.

LUPTON, Ellen (Org.). Tipos na Tela: um guia para designers, editores, tipógrafos, blogueiros e estudantes. São Paulo: Gustavo Gil, 2015.

MONTEIRO, Juliana Carolina Campos. A narrativa multilinear no livro digital educativo: uma experiência de desenvolvimento e utilização com alunos do $1^{\circ}$ ciclo do ensino básico. 2013. Dissertação - Mestrado em Multimídia, Universidade do Porto, Porto, 2013. Disponível: <http://bit.ly/MJCC2013>. Acesso: 5 nov. 2015.

\section{PINHEIRO, Tennyson; ALT, Luis. Design}

Thinking Brasil: empatia, colaboração e experimentação para pessoas, negócios e sociedade. Rio de Janeiro: Elsevier. 2011.

PUPPI, Maicon Bernet. Diretrizes para o design de interface de aplicativos para alemão como língua estrangeira via smartphones: um estudo sobre mobile learning. 2014. 206 f. Dissertação (Mestrado) - Curso de Programa de Pós-graduação em Design, Universidade Federal do Paraná, Curitiba, 2014.

ROGERS, Yvonne; SHARP, Helen; PREECE, Jennifer. Design de Interação: além da interaçãohumano-computador. 2 ed. Porto Alegre:

Bookman, 2013.

SAMARA, Timothy. Grid: construção e desconstrução. São Paulo: Cosac Naify, 2011. 


\section{$16^{\circ}$ \\ ERGODESIGN USIHC CINAHPA}

$16^{\circ}$ Ergodesign - Congresso Internacional de Ergonomia e Usabilidade de Interfaces Humano Tecnológica: Produto, Informações Ambientes Construídos e Transporte

$16^{\circ}$ USIHC - Congresso Internacional de Ergonomia e Usabilidade de Interfaces Humano Computador

CINAHPA | 2017 - Congresso Internacional de Ambientes Hipermídia para Aprendizagem.

SEHN, Thaís Cristina Martino. As possíveis configurações do livro nos suportes digitais.

2014. 272 f. Dissertação (Mestrado) - Programa de Pós-graduação em Comunicação e Informação, Universidade Federal do Rio Grande do Sul, Porto Alegre, 2014. Disponível em: <http://hdl.handle.net/10183/97246>. Acesso em: 05 nov. 2015.

SÍVERES, Luiz (Org.). A Extensão universitária como um princípio de aprendizagem. Brasília: Liber Livro, 2013.

SOUZA, Cejana Baiocchi (Org.). Temas contemporâneos em extensão das Instituições de Ensino Superior Comunitárias: Extensão, direitos humanos e formação da cidadania. Goiânia: Editora da PUC Goiás, 2014.

VIANNA, Maurício José; FILHO, Yasmar Vianna e Silva; ADLER, Isabel Krumholz; LUCENA, Brenda de Figueiredo; RUSSO, Beatriz. Design Thinking: inovação para negócios. Rio de Janeiro: MJV Press, 2012. 